\title{
Neuroendocrine tumor mixed with adenoma in rectum: a case report and literature review
}

\author{
Jiancong Hu, MD ${ }^{a, b}$, Xiaochuan Chen, MDc, Dezheng Lin, MD ${ }^{a, b}$, Zhaoliang Yu, MD ${ }^{b}$, Juan Li, MD ${ }^{a, b}$, \\ Xuefeng Guo, MD, PhD ${ }^{a, b *}$
}

\begin{abstract}
Neuroendocrine tumor (NET) mixed with adenoma in rectum is rare. A 63-year-old man with a rectal adenoma underwent endoscopic submucosal dissection. A $3.2 \mathrm{~cm}$ neoplasm was dissected, which histologically corresponded to a villous adenoma with high-grade intraepithelial neoplasia. More interestingly, cells arranged in strip were found in the submucosa, with SyN (+) and CgA $(-)$ in immunohistochemical staining, which were correspond to an NET. Although mixed adenoma with NET is rare, but we should alert it especially in large adenoma and full-scale preoperative evaluation should be taken before endoscopic operation.
\end{abstract}

Keywords: Adenoma, Neuroendocrine tumor, ESD

Neuroendocrine tumor (NET) is a distinct group of neoplasm that arises from enterochromaffin or neuroendocrine cells and display unique histomorphology. The NETs are composed of neuroendocrine secretory granules with neurosecretory capacity that can be detected by immunohistochemical staining. The NANETS Guidelines classification divided NET into low-grade (G1), intermediate-grade (G2), and high-grade $(\mathrm{G} 3)^{[1]}$. With the development of gastrointestinal endoscopy technology, most intestinal benign tumor can be resected under endoscopic surgery, such as adenoma or $\mathrm{NET}^{[2,3]}$.

The mixed adenoma with NET has been reported in only a few articles. All of the reported cases were found by postoperative pathology after enterectomy ${ }^{[4-6]}$. We present a rare case of NET mixed with a rectal adenoma dissected by endoscopic submucosal dissection (ESD), which was diagnosed by HE staining and immunohistologic staining.

\footnotetext{
a Department of Ambulatory Surgery Center, ' Guangdong Provincial Key Laboratory of Colorectal and Pelvic Floor Diseases, the Sixth Affiliated Hospital, Sun Yat-sen University and ${ }^{\circ}$ Department of Obstetrics and Gynecology, The First Affiliated Hospital of Sun Yat-sen University, Guangzhou, Guangdong, China

J.H. and X.C. contributed equally to this study and are co-first author.

The patient was informed consent.

This manuscript has been peer reviewed.

Sponsorships or competing interests that may be relevant to content are disclosed at the end of this article.

*Corresponding author. Address: The Sixth Affiliated Hospital, Sun Yat-sen University, 26 Yuancun Erheng Road, Guangzhou, Guangdong 510655, China. Tel.: +011-86-020-38254009; fax: 011-86-20-38254166. E-mail address: guoxf@126.com (X. Guo).

Copyright (C) 2019 The Authors. Published by Wolters Kluwer Health, Inc. on behalf of IJS Publishing Group Ltd. This is an open access article distributed under the terms of the Creative Commons Attribution-NonCommercial-ShareAlike 4.0 License,

which allows others to remix, tweak, and build upon the work non-commercially, as long as the author is credited and the new creations are licensed under the identical terms.

International Journal of Surgery Oncology (2019) 4:e79

Received 26 May 2019; Accepted 17 July 2019

Published online 29 October 2019

http://dx.doi.org/10.1097//J9.0000000000000079
}

\section{Case Report}

Two rectal polyps were found while a 63-year-old man underwent a routine colonoscopy examination. One was about $0.4 \times 0.8 \mathrm{~cm}$ and the other one was about $3.2 \times 0.8 \mathrm{~cm}$. Biopsy was performed in the larger lesion. The pathologic diagnosis was tubular adenoma. Blood test revealed CEA was slightly elevated at $8.58 \mathrm{ng} / \mathrm{mL}$. The patient was without other drug history, family history, and psychosocial history. Two polyps as shown in the former examination were found during the colonoscopy. The smaller polyp was removed by endoscopic mucosal resection. The larger polyp was elevated after submucosal injection and considered without submucosal invasion. ESD was performed and the neoplasm was dissected completely (Fig. 1). These procedures were performed by a junior resident with over 3 years of specialized training. The pathology diagnosis of the smaller one was villus-tubular adenoma. The large one was a villous adenoma with high-grade intraepithelial neoplasia with negative cutting edge. Interestingly, cells arranged in strip were found in the submucosa accidently. Mitosis was rare in the tumor cells ( $>2 / 10$ HPF). These cells were $\mathrm{SyN}(+)$ and $\mathrm{CgA}(-)$ by immunohistochemical staining and the proportion of $\mathrm{Ki}-67(+)$ cells of the tumor were about $2 \%$. Applied by the NANETS Guidelines ${ }^{[1]}$, it was diagnosed as a low-grade NET $(<2$ mitoses/10 HPF AND $<3 \% \mathrm{Ki}-67$ index) (Fig. 2). For further examination of residual or distant metastatic tumor, the patient underwent a CT scan after colonoscopic operation. No tumor residual and distant metastasis was found. The patient left with pleased and was discharged 6 days after colonoscopy without any complication.

\section{Discussion}

A mixed or collision tumor is composed of both adenocarcinoma and NET in a neoplasm. The 2 kinds of tumor originate from multipotent stem cells simultaneously differentiating into glandular and neuroendocrine lineages are plausible. This suggests that the 2 components of mixed tumors arise from multipotential stem cells and show bi-phenotypic differentiation ${ }^{[7]}$. Another hypothesis is that mixed tumor is a neuroendocrine phenotype of 

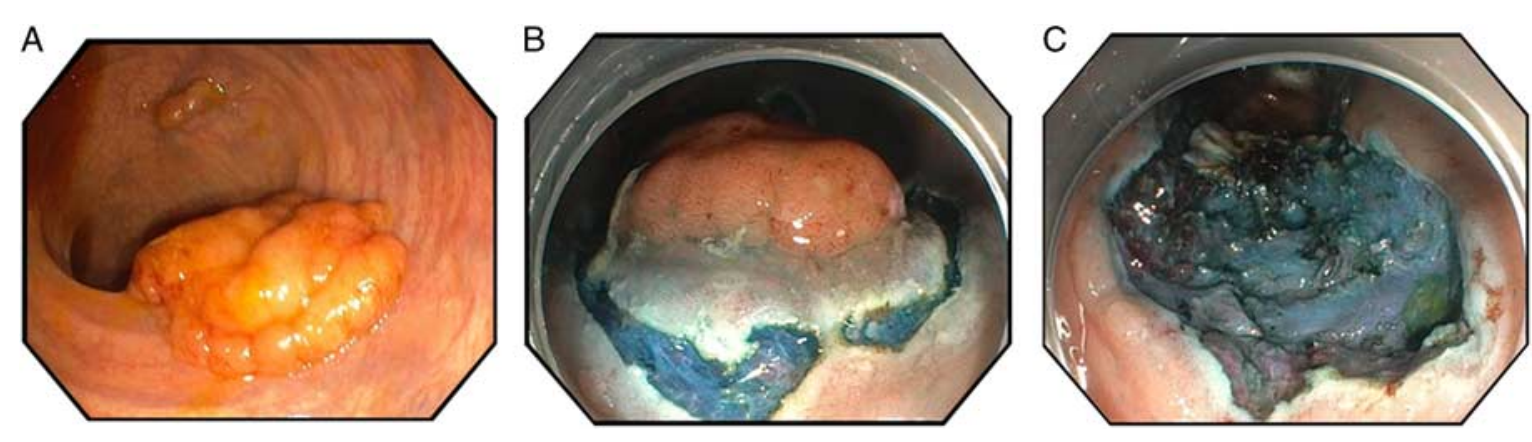

Figure 1. Endoscopic images and endoscopic submucosal dissection resection of the neoplasm (A-C).
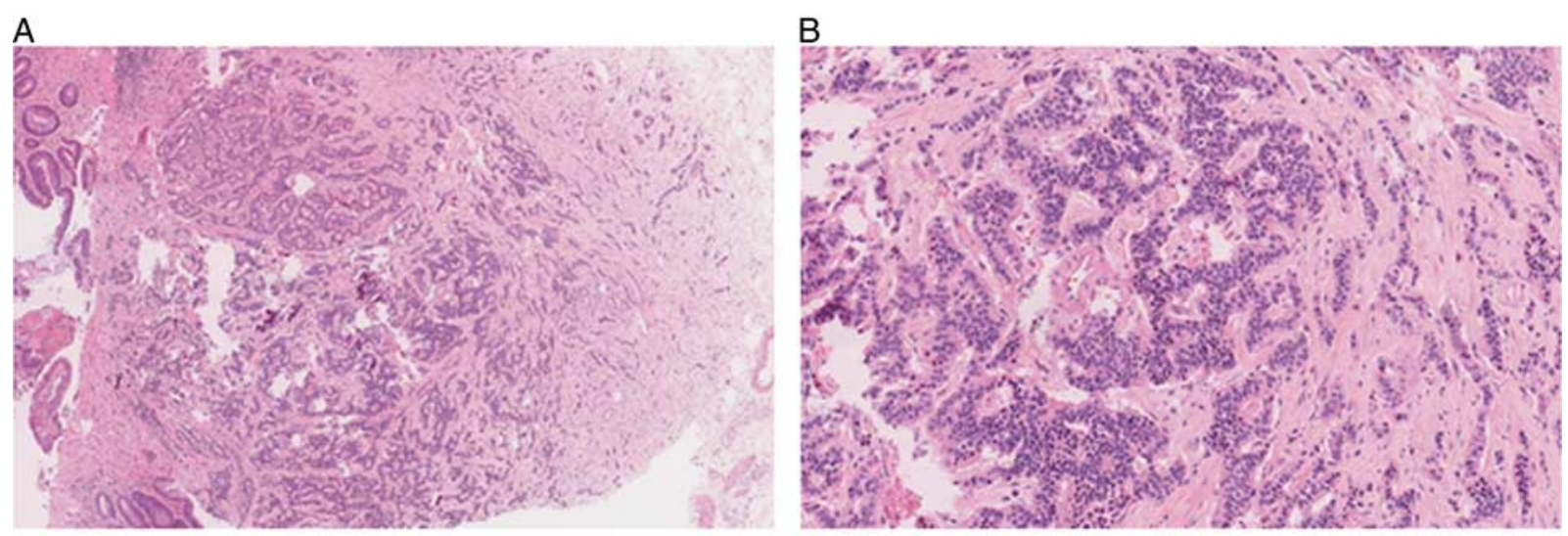

C
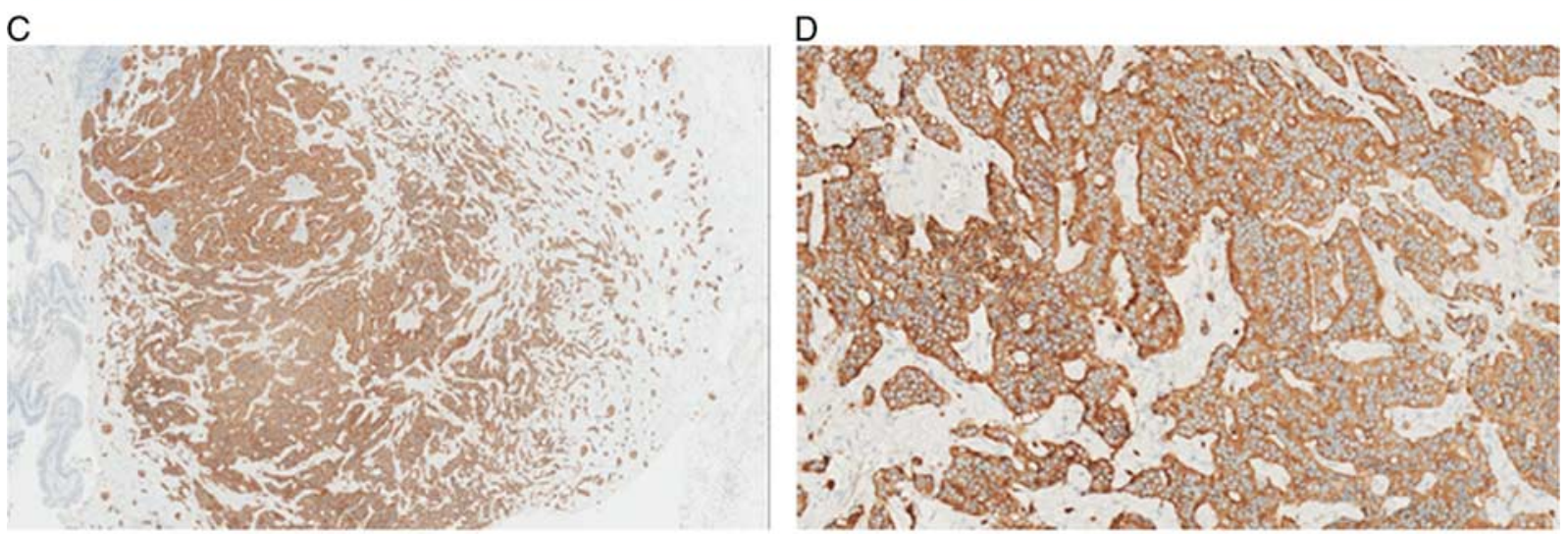

$\mathrm{E}$

$\mathrm{F}$

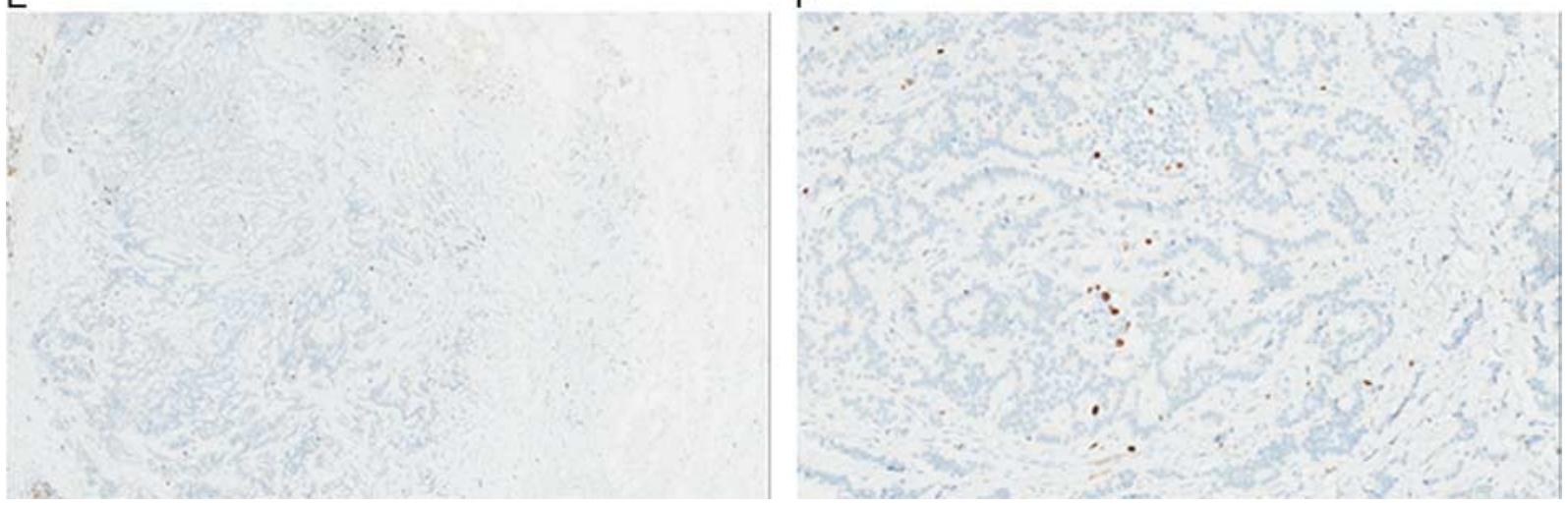

Figure 2. Pathologic section and immunohistochemically of the coexisting glandular-neuroendocrine neoplasm. A, HE staining $(\times 100)$. B, HE staining $(\times 400)$. C, Immunohistologic staining for SyN (x 100). D, Immunohistologic staining for SyN (× 400). E, Immunohistologic staining for Ki-67 (x 100). F, Immunohistologic staining for Ki-67 $(\times 400)$. 
Table 1

The literature review of cases with NET mixed with adenoma.

\begin{tabular}{|c|c|c|c|c|c|c|}
\hline No & Reference & Age (y) & Sex & Location & Nationality & Treatment \\
\hline 1 & Yamauchi et al[ ${ }^{[5]}$ & 41 & Male & Ascending colon & Japan & Right hemicolectomy \\
\hline 2 & Soliman et $\mathrm{al}^{[8]}$ & 68 & Male & Ascending colon & United States & Laparoscopic right hemicolectomy \\
\hline 3 & Kim et $\mathrm{al}^{[4]}$ & 70 & Male & Ascending colon & Korea & Right hemicolectomy \\
\hline 4 & Koletsa et al ${ }^{[9]}$ & 84 & Female & Sigmoid & Greece & Sigmoidectomy \\
\hline 5 & Lee et $\mathrm{al}^{[10]}$ & 56 & Female & Rectum & Korea & Low anterior resection \\
\hline 6 & Detweiler et al ${ }^{[11]}$ & 60 & Male & Colon & United States & Total proctocolectomy \\
\hline 7 & Gül-Klein et al ${ }^{[12]}$ & 65 & Female & Rectum & Germany & Hartmann procedure \\
\hline 8 & Gül-Klein et al ${ }^{[12]}$ & 63 & Male & Rectum & Germany & TaTME \\
\hline
\end{tabular}

TaTME indicates transanal total mesorectal excision.

dedifferentiated tubular adenocarcinoma ${ }^{[6]}$. A literature review conducted by Jessica N. Winn in 2017 summarized 17 cases of synchronous gastrointestinal carcinoid tumor and colon adenocarcinoma, but no literature review about collision tumor of NET and adenocarcinoma has been reported. Without this case, of the total 8 cases with NET mixed with adenoma in colon reported and we summarized them in Table 1.

Rectum is one of the most frequent lesion locations of digestive NET. Recent consensus guidelines on the management of rectal NET suggest that small tumors $(<1-2 \mathrm{~cm})$ confined to the mucosa or submucosa can be managed by endoscopic resection due to their low risk of metastatic spread ${ }^{[1]}$. Endoscopic ultrasonography is found to be useful for measuring the size and depth of rectal NET, which is essential for determining appropriate treatment ${ }^{[13]}$. In addition, the preoperative diagnosis of rectal NET may be more important due to its malignant potential.

ESD was recently developed for en bloc resection of large colorectal tumors and even early-stage gastrointestinal malignant neoplasms with negligible risk of lymph node metastasis ${ }^{[14,15]}$. In this case we resected the large adenoma by ESD. We found the adenoma was mixed of NET in the submucosa accidently. There were few reports of mixed glandular and $\mathrm{NET}^{[5,8]}$. All cases were treated with enterectomy and with high-grade malignancies. As we known, this is the first report of the 2 coexisting neoplasms resected by ESD.

Eggenberger ${ }^{[16]}$ reported that the resection of all visible primary disease was a reasonable treatment that offers the best prognosis for all midgut and hindgut NET. But NET may be mixed with adenoma and could be ignore by colonoscopy or biopsy. With the development of gastrointestinal endoscopy technology, more and more large adenoma could be resected by ESD. A risk of incomplete excision will occur in an adenoma mixed with NET. Once the metastasis of NET occurs, we currently have no standardized chemotherapy protocols that improve the patient's prognosis ${ }^{[16]}$. We need to be more vigilant to large adenoma before ESD. Full-scale preoperative evaluation should be taken before endoscopic operation. Endoscopic ultrasonography may be the appropriate choice. This work has been reported in line with the SCARE $2018^{[17]}$.

With the development of gastrointestinal endoscopy technology, large adenoma could be resected with ESD. The collision tumor of adenoma with NET is rare, but we should alert it especially in large adenoma and full-scale preoperative evaluation should be taken before endoscopic operation.

\section{Ethical approval}

This study was approved by the Ethical Committee of The Six Affiliated Hospital of Sun Yat-sen University.

\section{Sources of funding}

None.

\section{Author contribution}

J.H., D.L., X.C. made substantial contributions to the study conception, acquisition of data, analysis of data, data interpretation, and draft the manuscript. Z.Y., J.L., X.G. made contributions to the study conception, analysis of data, and data interpretation.

\section{Conflict of interest disclosure}

The authors declare that they have no financial conflict of interest with regard to the content of this report.

\section{Research registration unique identifying number (UIN)}

None.

\section{Guarantor}

None.

\section{Acknowledgment}

The authors thank Yan Huang, Department of Pathology, the Sixth Affiliated Hospital of Sun Yat-sen University for providing the pathologic data.

\section{References}

[1] Anthony LB JRS, Klimstra DS, Maples WJ, et al. The NANETS Consensus Guidelines for the Diagnosis and Management of Gastrointestinal Neuroendocrine Tumors (NETs) Well-Differentiated NETs of the Distal Colon and Rectum. Pancreas 2010;39:767-4.

[2] Kim J, Kim JH, Lee JY, et al. Clinical outcomes of endoscopic mucosal resection for rectal neuroendocrine tumor. BMC Gastroenterol 2018; $18: 77$. 
[3] Samalavicius NE, Smolskas E, Mikelis K, et al. Transanal endoscopic microsurgery for rectal adenomas: single center experience. Wideochir Inne Tech Maloinwazyjne 2016;11:26-30.

[4] Kim YN, Park HS, Jang KY, et al. Concurrent large cell neuroendocrine carcinoma and adenocarcinoma of the ascending colon: a case report. J Korean Soc Coloproctol 2011;27:157-61.

[5] Yamauchi H, Sakurai S, Tsukagoshi R, et al. A case of very well-differentiated adenocarcinoma with carcinoid tumor in the ascending colon. Int Surg 2014;99:132-6.

[6] Gurzu S, Kadar Z, Bara T, et al. Mixed adenoneuroendocrine carcinoma of gastrointestinal tract: report of two cases. World J Gastroenterol 2015;21:1329-33.

[7] Kitajima T, Kaida S, Lee S, et al. Mixed adeno(neuro)endocrine carcinoma arising from the ectopic gastric mucosa of the upper thoracic esophagus. World J Surg Oncol 2013;11:218.

[8] Soliman ML, Tiwari A, Zhao Q. Coexisting tubular adenoma with a neuroendocrine carcinoma of colon allowing early surgical intervention and implicating a shared stem cell origin. World J Gastroenterol 2017;23:1106-2.

[9] Koletsa TBE, Tziola T, Mavropoulou S, et al. Neuroendocrine carcinoma in adenoma of the sigmoid. Hippokratia 2014;18:362-3.

[10] Lee SY, Hwang DY, Hwang TS, et al. Neuroendocrine dysplasia combined in a tubular adenoma of rectum: a case report. Korean J Pathol 2013;47:495-8.
[11] Detweiler CJ, Cardona DM, Hsu DS, et al. Primary high-grade neuroendocrine carcinoma emerging from an adenomatous polyp in the setting of familial adenomatous polyposis. BMJ Case Rep 2016;2016: bcr2015214206.

[12] Gul-Klein S, Sinn M, Jurmeister PS, et al. Two patients with rare mixed adenoneuroendocrine carcinomas of the rectum. SAGE Open Med Case Rep 2018;6:2050313X18758816.

[13] Ishii N, Horiki N, Itoh T, et al. Endoscopic submucosal dissection and preoperative assessment with endoscopic ultrasonography for the treatment of rectal carcinoid tumors. Surg Endosc 2010;24:1413-9.

[14] Yamamoto K, Michida T, Nishida T, et al. Colorectal endoscopic submucosal dissection: recent technical advances for safe and successful procedures. World J Gastrointest Endosc 2015;7:1114-28.

[15] Colak S, Gurbulak B, Cakar E, et al. Evaluation of endoscopic mucosal resection and endoscopic submucosal dissection in submucosal lesions of the colon and rectum. Wideochir Inne Tech Maloinwazyjne 2018;13: 448-53.

[16] Eggenberger JC. Carcinoid and other neuroendocrine tumors of the colon and rectum. Clin Colon Rectal Surg 2011;24:129-34.

[17] Agha RA, Borrelli MR, Farwana R, et al. The SCARE 2018 statement: updating consensus Surgical CAse REport (SCARE) guidelines. Int J Surg 2018;60:132-6. 